\title{
EDITORIAL
}

\section{The neurobiological basis of psycho-oncology}

\author{
Juliana Augusta Garcia, ${ }^{1,2}$ Maria Teresa Lourenço, ${ }^{1,2}$ Jair de Jesus Mari ${ }^{3}$ \\ ${ }^{1}$ Núcleo de Psico-Oncologia, AC Camargo Cancer Center, São Paulo, SP, Brazil. ${ }^{2}$ Centro de Oncologia, Hospital Alemão Oswaldo Cruz, \\ São Paulo, SP, Brazil. ${ }^{3}$ Departamento de Psiquiatria, Universidade Federal de São Paulo (UNIFESP), São Paulo, SP, Brazil.
}

Several studies have sought to investigate whether changes in the ability of the immune response to fight malignant cells could be associated with onset or progression of cancer, and have linked stress, depression, and lack of psychosocial support with malignancies. ${ }^{1}$ Cancer is an umbrella term that refers to a range of chronic diseases characterized by uncontrolled growth of cells that invade tissues and organs and can spread to other parts of the body. This process has a strong biological basis and is modulated by psychosocial factors. As do most psychiatric disorders, cancer has a multifactorial etiology, including genetic, biological, and environmental components.

Stress is understood as the experience of a negative life event or the occurrence of an event that generates subjective feelings of inadequacy in coping effectiveness. The brain is the central organ of stress and adaptation. While acute stress is a normal response associated with physiological changes necessary to body homeostasis, chronic stress can be linked to maladaptive brain changes and a predisposition to development of several diseases. ${ }^{2}$ A diagnosis of cancer is usually associated with greater chronic psychological stress than non-neoplastic malignant diseases with similar prognosis.

Psychoendoneuroimmunology studies have demonstrated the interrelationships between psychological (mental) and biological (central nervous system, immune, and endocrine) processes. This integration assumes that the neuroendocrine and immune systems share receptors and substances with modulating effects on immune events. In chronic stress, neuroendocrine activation is represented by stimulation of the sympathetic nervous system (the main neurotransmitters of which are adrenaline and noradrenaline) and of the hypothalamic-pituitary-adrenal axis, with consequent increases in glucocorticoid expression. This activation, combined with tissue damage resulting from the malignant neoplasm itself and from interventions used in cancer treatment (chemotherapy, radiotherapy, and surgery), is associated with a number of changes observed in the tumor microenvironment, with consequent stimulation of local inflammatory response. ${ }^{1,3}$

This increase in local inflammation, represented principally by elevated production of cytokines such as interleukin 1 (IL-1), IL-6, interferon alpha, and tumor necrosis factor

Correspondence: Juliana Augusta Garcia, Rua Borges Lagoa, 564, conjunto 23, CEP 04038-000, São Paulo, SP.

E-mail: juliplens@gmail.com alpha (TNF- $\alpha$ ), will promote a series of cellular and molecular changes. These events impair regulation of the immune response, which could enable activation of oncogenic viruses, alterations in immune cell (including T-lymphocyte and natural killer cell) response, and promote tumor cell survival, thus facilitating metastasis through modulation of angiogenesis, proliferation, invasion, embolization, and colonization of remote sites by malignant cells. Such effects are modulated by a variety of neurotransmitter receptors found in leukocytes, stromal cells, and tumor cells. ${ }^{1}$

Several studies show that the neurotransmitter serotonin plays important roles in immune regulation, as a series of immune system cells express serotonin receptors. It is therefore expected that pharmacological modulation of the serotonin system would be associated with immunomodulatory effects, which could be related to a change in progression of inflammation-based diseases such as cancer. Neutrophil and macrophage chemotaxis, migration of dendritic cells, antigen presentation with subsequent generation of immune memory (innate and acquired immune response), phagocytosis, opsonization, decreased TNF- $\alpha$ and IL-12, IL-1, increased IL-6, IL-8, and IL-10, cellular differentiation (monocytes, macrophages), and apoptosis are all events that are modulated to some extent by serotonergic transmission. ${ }^{4}$

Depressive symptoms are three times more prevalent in people with cancer than expected in the general population, with the highest rates found near the time of diagnosis. This higher prevalence is due to a number of predisposing factors, not only demographic and medical, but also those associated with the underlying disease and its inflammatory process, which can be associated both with the pathophysiology of depression and with tumor growth. ${ }^{5}$ There is a two-way relationship between stress/ chronic depression and inflammation; stress interferes with pathophysiological mechanisms associated with inflammation, while the inflammatory exacerbation site acts as a stimulus to the central nervous system, providing feedback and impairing serotonergic and dopaminergic transmission. ${ }^{3}$ These neurotransmitter changes predispose patients to a depressive state, with a continuum of symptoms ranging from non-pathological sadness, through adjustment disorder with predominant depressive symptoms, to frank depressive disorder. ${ }^{5}$ Fatigue and energy loss are common symptoms, and are important components of sickness behavior in these patients. 
Currently, it is acknowledged that chronic stress and depression have a negative impact on patients with cancer, mainly affecting their quality of life and disease progression. Depression is an independent predictor of worse prognosis and poor quality of life in these patients; it is associated with impaired coping with the disease, poorer treatment adherence, faster progression to advanced cancer, longer hospital stays, worse depressive symptoms during chemotherapy in untreated patients, and higher mortality, including by suicide.

It is well known that combined pharmacotherapeutic and psychosocial interventions can alleviate the stress response, enhance quality of life, and improve prognosis. ${ }^{5}$ Selective serotonin reuptake inhibitors are drugs of choice in this patient population, given their potential antiinflammatory (microglia), antitumor (apoptosis), and immunomodulatory properties, but the clinical significance of these immunological and cellular effects is still unclear. ${ }^{6}$

An individualized and integrated approach that addresses organic and psychosocial dimensions is particularly important when treating patients with malignancies. ${ }^{5}$ However, data are still lacking to support more effective management strategies. Topics for investigation include drugs with cellular and molecular targets that modulate inflammation, ${ }^{1}$ establishing which non-pharmacological interventions are most effective, and determining the most opportune time for implementation of such interventions. ${ }^{5}$ Psycho-oncology is a vast, incipient field for future research.

\section{Disclosure}

The other authors report no conflicts of interest.

\section{References}

1 Currier MB, Nemeroff CB. Depression as a risk factor for cancer: from pathophysiological advances to treatment implications. Annu Rev Med. 2014;65:203-21.

2 McEwen BS. Brain on stress: How the enviroment gets under the skin. Proc Natl Acad Sci USA. 2012;109:17180-5.

3 Miller AH, Ancoli-Israel S, Bower JE, Capuron L, Irwin MR. Neuroendocrine-immune mechanisms of behavioral comorbidities in patients with cancer. J Clin Oncol. 2008;26:971-82.

4 Arreola R, Beccerril-Villanueva E, Cruz-Fuentes C, Velasco-Vélasquez MA, Garcés-Alvarez ME, Hurtado-Alvarado G, et al. Immunomodulatory effects mediated by serotonin. J Immunol Res. 2015;2015: 354957.

5 Li M, Fitzgeral P, Rodin G. Evidence-based treatment of depression in patients with cancer. J Clin Oncol. 2012;30:1187-96.

6 Thekid SM, Trinidad A, Roth A. Psychopharmacology in cancer. Curr Psychiatry Rep. 2015;17:529. 